\title{
Selbsterfahrung in der Psychodrama Ausbildung
}

\author{
Jutta Fürst
}

Angenommen: 20. Januar 2022 / Online publiziert: 17. Februar 2022

(C) Der/die Autor(en) 2022

Zusammenfassung In diesem Beitrag der Zeitschrift für Psychodrama und Soziometrie wird erörtert, dass Selbsterfahrung in der Ausbildung zur Psychodramatherapeut*in im Vergleich zur Selbsterfahrung in anderen psychotherapeutischen Ansätzen, aus historischen, philosophischen und technischen Gründen eine besondere Form hat. Der wissenschaftlichen Auseinandersetzung mit der Selbsterfahrung wurde in anderen therapeutischen Verfahren ungleich mehr Raum gegeben als im Psychodrama. Auch die Anforderungen an die Ausbildung haben sich in den letzten Jahren besonders durch die Akademisierung geändert.

Aus diesen Gründen ist es mehr als sinnvoll, die Entwicklung der Selbsterfahrung als Element der Ausbildung näher zu betrachten, um aus den bisherigen Erfahrungen, Perspektiven für eine zukünftige Ausbildungsgestaltung zu erarbeiten.

Der Beitrag versucht die Unterschiede sichtbar zu machen und die Entwicklung der Selbsterfahrung als Ausbildungsteil nachzuzeichnen. Dabei werden die Ziele, Wirkweise sowie Vor- und Nachteile der Selbsterfahrung in der Ausbildung beschrieben, Rückschlüsse aus den bisherigen Erfahrungen gezogen und mögliche Änderungen für die Zukunft skizziert.

Schlüsselwörter Selbsterfahrung $\cdot$ Psychodrama $\cdot$ Ausbildung

Jutta Fürst $(\bowtie)$

Schumacherweg 6/I, 6060 Hall, Österreich

E-Mail: jutta.fuerst@cnh.at

Sigmund Freud Privatuniversität Wien, Wien, Österreich 


\section{Self-awareness training in psychodrama}

Abstract In this article of the Zeitschrift für Psychodrama und Soziometrie it is discussed that caused by historical, philosophical and technical reasons, selfawareness-training in psychodrama education takes on a unique form in comparison to other psychotherapeutic approaches. In relation to other modalities there is still a lack of scientific discussion regarding concepts of self-awareness in psychodrama training. The requirements for training have also changed in recent years, particularly as a result of academization.

Therefore, it is more than reasonable to look closer at the development of selfawareness therapy in training. Taking research and practical experiences into consideration it is possible to create better training for the future.

This contribution shows the development of self-awareness training as a part of the general psychodrama education. The goals, mode of action, and advantages and disadvantages of self-awareness in training are described, conclusions are drawn from past experience, and possible changes for the future are outlined.

Keywords Self-awareness-training $\cdot$ Psychodrama $\cdot$ Training

\section{Einleitung}

Nach über 45 Jahren persönlicher Erfahrung in verschiedenen Ausbildungen, Praxis und Lehre, in denen Selbsterfahrung (SE) inkludiert waren, stellt sich dieser ZPS Schwerpunkt als günstige Gelegenheit dar, der Frage nachzugehen, was SE in der Psychodramaausbildung von anderen Modalitäten unterscheidet, was sie zur Qualitätssicherung leistet und in welchen Bereichen es sinnvoll wäre, Struktur und Methodik zu verändern.

Basierend auf der Annahme, dass psychische Störungen, aber auch Probleme im Berufsalltag und im Leben generell immer auch Beziehungsstörungen sind und eine gelungene Therapeut*in-Patient*in-Beziehung einen wesentlichen Wirkfaktor (Bergin und Garfield 1971) darstellt, muss die Auseinandersetzung von angehenden Psychotherapeut*innen, Berater*innen und Supervisor*innen mit ihren Beziehungserfahrungen und Beziehungsmustern einen wesentlichen Teil des Trainings bilden.

Diese Auseinandersetzung soll verhindern, dass in der therapeutischen bzw. beratenden Situation bei den Therapeut*innen unbekannte Gefühle und Reaktionen angeregt werden, die eine förderliche Beziehungsgestaltung stören bzw. verhindern. Im Gegenteil dazu sollen die, in der Ausbildung gemachten Erfahrungen bezüglich persönlicher Kränkungen, Bedrohungen und Unzulänglichkeiten in der eigenen Lebensgeschichte dazu beitragen, eine förderliche, schützende und unterstützende Haltung beizubehalten.

Dieser Ansatz wird jedoch durchaus kontrovers diskutiert. Früher, aber auch heute noch, sind manche Verhaltenstherapeut*innen gegen eine SE in der Ausbildung, weil die Sinnhaftigkeit als nicht bestätigt gilt (Tschuschke 2016), während für die Psychoanalyse seit jeher die intensive Lehranalyse Voraussetzung für jeden Psychoanalytiker und jede Psychoanalytikerin war. 
Die Sichtweise, dass SE zur Qualität der Psychotherapie und ähnlicher Formate einen wesentlichen Beitrag leistet, wird auch von den Gesetzgebern jener Länder geteilt, in denen Psychotherapie als eigener Berufsstand anerkannt und sowohl Ausbildung als auch Tätigkeit staatlich kontrolliert wird.

\section{Selbsterfahrung im Psychodrama}

Psychodrama nimmt im Gegensatz zu anderen Verfahren einen besonderen Standpunkt ein. Historisch betrachtet war Psychodrama in seiner Anfangsphase nicht auf Heilung psychischer Störungen angelegt, sondern auf Entwicklung persönlicher und zwischenmenschlicher Potenziale (Moreno 1974). Mit dem Ziel eine therapeutische Weltordnung zu schaffen, stellte Moreno sich außerhalb gezielt agierender professioneller Gruppen aus dem Gesundheitsbereich. Psychodrama wurde deshalb oft mehr als Lebenshaltung betrachtet und in Therapie, Erziehung, Beratung, Lehre, Management, Theater u.a. gleichermaßen eingesetzt. Es ist besonders Ferdinand Buer (1999) zu verdanken, dass er auf die notwendige Trennung von Verfahren und Format hingewiesen hat. Nur so ist es möglich, dass Psychodrama als Verfahren in verschiedenen Formaten, wie Psychotherapie, Supervision, Beratung und Coaching Anerkennung erfährt.

Die grundsätzliche psychodramatische Annahme, dass der Mensch von Natur aus kreativ und zur spontanen Handlung fähig ist und diese Ressource nur zur Entfaltung gebracht werden muss, um Lösungen für verschiedenste Probleme zu finden, blieb auch später, als Psychodrama längst als psychotherapeutisches Verfahren eingesetzt wurde, erhalten. Die formatspezifische Weiterentwicklung in Praxis und Lehre wurde dadurch hintangehalten. Ausbildungen für verschiedene Berufe wurden vermischt, Curricula nicht präzisiert, Ziele nicht definiert und Ergebnisse nicht überprüft.

Die Auseinandersetzung mit gesetzlichen Anforderungen, anderen psychotherapeutischen Verfahren, der Positionierung in der Wissenschaft und der allgemeinen Akademisierung verschiedener Berufszweige hat auch das Psychodrama als Verfahren gezwungen, sich den Erfordernissen anzupassen.

In der Ausbildung zum „Psychodramatiker bzw. Psychodramatikerin“, unabhängig von jedem Format, wurde dem Beziehungsgeschehen immer schon eine zentrale Bedeutung zugemessen. Die Exploration der persönlichen und kollektiven Beziehungsgeschichte, sowie die Entwicklung von Kreativität und Spontaneität standen seit den Anfangszeiten im Mittelpunkt.

Diese Auseinandersetzung schließt das Wiedererleben verstörender, wie gelungener Interaktionen ein, um ungeeignete wiederkehrende Handlungs- und Erlebensmuster zu erkennen und neue Varianten zu ermöglichen.

\section{Definition und Begrifflichkeit}

Der Begriff „Selbsterfahrung“ wird im deutschen Sprachraum in den meisten Therapieverfahren für jenen Bereich der Ausbildung verwendet, der der Persönlichkeitsentwicklung, dem sozialen Lernen und der Selbstreflexion dient. Im psycho- 
analytischen Verfahren wird nach wie vor von Lehranalyse gesprochen. Auch der Begriff Lehrtherapie findet gelegentlich Anwendung. Im englischen Sprachraum gibt es keinen einheitlichen Begriff dafür. „Personal-development“, ,,personal-therapy“ oder "self-awareness-training“ sind am gebräuchlichsten. Das österreichische Psychotherapiegesetz schreibt: „Die Bezeichnung Lehrtherapie, Lehranalyse, Gruppenund Einzelselbsterfahrung sind als synonyme Begriffe zu verstehen“ (Kierein et al. 1991).

\section{Historische Entwicklung}

Die Bedeutung der Gruppe als Ort und Möglichkeit sich mit sich selbst und anderen auseinanderzusetzen, wurde von verschiedenen Therapeut*innen in der Zeit des 2. Weltkrieges und danach betont. Besonders zu erwähnen sind hier Samuel Slavson, Jakob L. Moreno, Kurt Lewin, Wilfred R. Bion, Sigmund H. Foulks und Carl R. Rogers. Mit Ausnahme von Moreno stammten ihre Vorerfahrungen alle aus dem Einzelsetting der eigenen Lehranalyse.

Moreno hat den Begriff SE in der Ausbildung nie explizit erwähnt. Psychodramagruppen waren von Beginn an, als ,learning by doing“ angelegt. Menschen explorierten ihre Ängste, Wünsche, Erlebnisse, Träume auf der Bühne, andere Gruppenteilnehmer*innen übernahmen die ihnen zugeteilten Rollen und der Leiter bzw. die Leiter*in schuf Struktur und regelte den Ablauf und den Einsatz der Techniken.

Die damit stattfindende SE war weder zielgerichtet noch systematisch. Buer (2010, S. 247) formuliert es so: „Moreno hatte nämlich keine Lehre zu verkünden. Er bot stattdessen ,symbolische Behälter' an, in denen sich Menschen begegnen können, die daran interessiert sind, ihre Lebens- und Arbeitsverhältnisse gemeinsam zu untersuchen, sich auf einen persönlichen und sozialen Wandlungsprozess einzulassen, unkonventionelle Erkenntnisse zu gewinnen, kreative Lösungen zu erfinden und solidarisch an ihrer Verwirklichung zu arbeiten“.

Deshalb wurde auch in den Anfängen der institutionalisierten psychodramatischen Ausbildung nicht von Selbsterfahrungstraining gesprochen, sondern vielfach von fortlaufenden Psychodrama-Gruppen, die die Möglichkeit boten, alle auftauchenden Themen in der Gruppe zu explorieren.

In den 70er-Jahren erlebte die SE in Gruppen durch „Sensitivity-Trainings“ und „Encounter-Gruppen“ einen Höhepunkt, der in den folgenden Jahrzehnten wieder abflachte.

In der Verhaltenstherapie und systemischen Therapie, in der der richtigen Anwendung der Interventionen lange Zeit mehr Bedeutung beigemessen wurde, als der Therapeut*innenpersönlichkeit und dem Beziehungsaspekt, wurde die SE erst dann als sinnvoll betrachtet, als wissenschaftliche Studien nahelegten, dass die Therapeut*in-Patient*in-Beziehung einen wichtigen Wirkfaktor darstellt.

In der Psychoanalyse fand die SE bzw. Lehranalyse in einem individuellen Setting statt, während andere Verfahren, auch als Folge der Sensitivity-Trainings in den 70er-Jahren, die SE mit einigen Ausnahmen, auch aus ökonomischen Gründen, in die Gruppe verlegten. 
Im Gegensatz zum Psychodrama blieb es aber überwiegend eine individuelle Arbeit an persönlichen Themen im Rahmen der Gruppe. Moreno betrachtete die Gruppe hingegen als Abbild der Gesellschaft und als ideales Umfeld, um neue Dimensionen der Psyche zu eröffnen und zu erforschen (Hutter und Schwehm 2009). Grete Leutz zitiert Moreno wenn sie schreibt: „Psychodrama ist Therapie in der Gruppe, durch die Gruppe, für die Gruppe und der Gruppe“ (Leutz 1974, S. 92). Diese Sichtweise gibt der Gruppe im Psychodrama ungleich mehr Bedeutung als anderen Modalitäten. Auch im Einzelsetting werden Gruppen, an denen jemand teilhat, in den Prozess immer einbezogen.

\section{SE als Ausbildungsteil der Psychotherapieausbildung}

In der Psychoanalyse wird die Bewusstmachung unbewusster Inhalte als Form der Heilung betrachtet. Deshalb wurden „Ausbildungsteilnehmer*innen zunächst jener ,psychoanalytischen Kur' ausgesetzt, die sie später bei ihren PatientInnen selbst durchführen sollten“" (Mösler et al. 2016).

Lange Zeit wurde davon ausgegangen, dass Psychotherapie und im Besonderen eine Psychoanalyse nur im Zweiersetting möglich sei. Es war ein Verdienst Morenos, die Wirkkraft der Beziehungen und der Gruppe in den Mittelpunkt zu stellen. Er forschte und publizierte bereits zur Wirkweise sozialer Beziehungen (Moreno 1945; Moreno und Toeman 1942) als Foulkes (Foulkes und Anthony 1984) und Balint (Balint und Balint 1955) in England, sowie Lewin (Moreno 1953) und Rogers (1977) in den USA erst begannen die Gruppe in den Mittelpunkt zu rücken.

Die Wendung vom Individuellen hin zur Gruppe fand seinen Höhepunkt in den 60er und 70er-Jahren des vorigen Jahrhunderts. Encounter-Gruppen und SensitivityTraining wurden zum Experimentierfeld für Forscher*innen, Studierende und Psychotherapeut*innen. In diesen Encounter-Gruppen und Sensitivity-Trainings fand keine systematische Reflexion der persönlichen Innenwelt statt. Auch wenn diese, je nach theoretischer Orientierung der Leiter*in mehr oder weniger strukturiert abliefen, bezogen sie ihre Attraktion aus der Möglichkeit, Erfahrungen im zwischenmenschlichen Bereich in einem mehr oder weniger geschützten Rahmen zu machen.

Psychodramatische Gruppen folgten hingegen einer klaren Abfolge von Erwärmung, Inszenierung und Reflexion. Damit war ein Wechsel von Aktionserfahrung und intellektueller Verarbeitung gewährleistet. Die Szenen waren entweder personen- oder gruppenzentriert. Die Gruppe und die darin gesammelten Beziehungen und Rollen hatten aber im Gegensatz zu anderen therapeutischen Verfahren, wie z.B. in der Gestalttherapie, eine ungleich höhere Bedeutung.

Die frühen Ausbildungsgruppen Morenos folgten demselben Konzept wie die Patient*innengruppen. Das Thema, dem die Gruppe ihren Vorzug gab, wurde zur Bearbeitung gewählt. Die Themen folgten der Dynamik der Gruppe.

Auch in den Ausbildungsgruppen, in den von seinen Schüler*innen gegründeten Ausbildungsinstituten, wurde ähnlich gearbeitet. In den 70er-Jahren wurden mehr und mehr didaktisch-methodische Überlegungen angestellt und die Ausbildung verändert. SE wurde von Theorieseminaren stundenmäßig getrennt, wobei die Theorie 
vorrangig darin bestand, dass ein bestimmtes Thema in den Mittelpunkt gestellt wurde, aber dennoch in derselben Weise psychodramatisch, erfahrungsorientiert gearbeitet wurde. Diese Gruppen unterschieden sich nur durch den Themenschwerpunkt von SE-Gruppen.

Die sogenannten „Oberstufen“ oder ,,advanced-groups“ waren Psychodramagruppen, in denen Auszubildende Leiter*innenfunktion übernahmen, um ihr Handwerk zu lernen. Nach wie vor wurde in diesen Gruppen protagonisten- und gruppenzentriert gearbeitet. Dadurch war der Anteil an SE gegenüber Theorie und Technik sehr hoch. Die Vermischung von Theorie, SE und Techniklehre führte zu Irritationen und Ängsten bei den Teilnehmer*innen. Die Leiter*innen nahmen zwangsläufig Mehrfachrollen ein. Sie waren Therapeut*innen, Referent*innen, Supervisor*innen und Prüfer*innen in einer Person.

Die Auszubildenden waren „Patient*innen“ und Studierende zum selben Zeitpunkt. Sie sollten sich öffnen und ihr Selbst explorieren und fühlten sich gleichzeitig bewertet und kritisiert. Dass dieses Gefühl in früheren Ausbildungen kaum bis gar nicht existierte, scheint der immer stärkeren Verschulung der Ausbildung geschuldet.

Auch in der Praktikums- und Praxissupervision ist die Vorgangsweise nicht einheitlich. In einigen Ausbildungseinrichtungen war und ist Supervision mit SE eng verbunden, in manchen weitgehend getrennt (Krall et al. 2013). Einige Supervisor*innen vermeiden die psychodramatische Arbeit in der Supervision, um SE möglichst auszuschließen und damit Funktionsüberschneidungen zu vermeiden. Pierre Fontaine (Fontaine 1994) sammelte die Daten von 15 Ausbildungsinstituten in 15 verschiedenen Ländern. In einer Übersicht stellte er das stundenmäßige Ausmaß von SE, Theorie und Methodik in den einzelnen Ausbildungen dar. Durch die Vermischung von SE, Theorie und Technik war es den Ausbildungsinstituten jedoch kaum möglich, eine stundenmäßige genaue Trennung der Teilbereiche vorzunehmen.

In den meisten Ausbildungseinrichtungen wurde unter SE jener Teil der Gruppenarbeit verstanden, der ,,dem persönlichen Wachstum diente“. Eine genauere Beschreibung bzw. Operationalisierung fehlte. Deshalb sind die gesammelten Daten auch nur vorsichtig interpretierbar. Die Gesamtstundenzahl der Ausbildung reichte von 240-1900h, der Anteil der ausgewiesenen SE wurde mit 200-500h angegeben (Fontaine 1994).

Die Ausbildungen unterschieden sich aber auch in den Voraussetzungen. Manche Institute verlangten SE vor Beginn der Ausbildung. Bei vielen war es Teil der Ausbildung selbst. Auch die Ziele waren nicht dieselben. Die einen bildeten ausschließlich Psychotherapeut*innen aus. Andere zielten auf Fachpersonal im pädagogischen oder organisatorischen Bereich ab. Wieder andere vermischten beides.

Während die Methodik und Didaktik von Theorievermittlung und SE in den meisten Modalitäten deutlich voneinander abgrenzbar ist, war und ist sie in der Psychodramaausbildung durchwegs verwoben.

Theorie wird anhand von praktischen, handlungsorientierten Übungen vermittelt, die zwangsläufig eine Erfahrung mit sich selbst einschließen. Dies erfolgt aus einer psychodramatheoretischen Überlegung. Kognitiver Wissenserwerb braucht in Folge dieser Annahme, um wirklich verstanden und verankert zu werden, die Erfahrung über alle Sinneseindrücke und die Verbindung mit dem Gefühl und persönlichen 
Erlebnissen. Lernen aus psychodramatischer Sicht ist immer erfahrungsorientiert und schließt aus pädagogischer Sicht an die Erkenntnisse des entdeckungsorientierten Lernens nach Piaget (Scholkmann 2016) und Bruner (Bender 2003) an.

Das bedeutet, dass sich die psychodramatische Arbeitsweise mit ihrem lehrimmanenten Selbsterfahrungsaspekt durch alle Ausbildungsteile erstreckt. Auch die Supervision (Krall et al. 2013) erfolgt mehrheitlich mit Hilfe psychodramatischer Techniken, die Inszenierung, Rollentausch u. a. einschließt.

SE findet in österreichischen Ausbildungseinrichtungen zum Teil im Einzelsetting statt. Die minimale Stundenanzahl variiert von 50 bis $100 \mathrm{~h}$. Diese Entwicklung resultierte aus der Erfahrung, dass Psychotherapeut*innen, aber auch Berater*innen und Supervisor*innen, auch oder ausschließlich mit einzelnen Klient*innen arbeiten und der Therapieverlauf in einem Einzelsetting besser anhand eines Selbsterfahrungsprozesses erfahren werden kann.

\section{Ziele}

Da in der Verhaltenstherapie der Therapeut*innenpersönlichkeit erst Aufmerksamkeit geschenkt wurde, als der wissenschaftliche Nachweis erfolgte, dass die Therapeut*in-Patient*in-Beziehung ein wesentlicher Wirkfaktor ist, ist die Auseinandersetzung mit der SE, ihren Zielen und der didaktisch-methodischen Umsetzung in diesem Verfahren sehr jung.

Die Ziele in den meisten anderen Therapieansätzen sind eher vage definiert. So soll SE grob gesagt, die therapeutische Kompetenz der angehenden Psychotherapeut*innen verbessern (Schön 2001).

Die klientenzentrierte Gesprächstherapie nach Rogers sieht das Ziel weniger im Veränderungsprozess als mehr im Erkenntnisgewinn. Dabei steht die Einfühlung in die Rolle des Patienten bzw. der Patientin, indem man selbst „Patient*in“ in diesem dyadischen Geschehen der Lehrtherapie ist, sowie das Lernen am Modell und im Besonderen das Erleben, im Vordergrund (Korbei 1996).

Nodop und Strauß (2016, S. 58) definieren das Ziel der SE allgemein, indem das Ergebnis eine Psychotherapeut*innen-Persönlichkeit sein soll, ,die fähig ist, persönliche Voraussetzungen für das therapeutische Erleben und Handeln zu reflektieren und zu modifizieren".

Schmelzer und Hirsch (2000) sieht als Ziel einerseits die Begrenzung von Risiken, die durch den Therapeuten bzw. die Therapeutin entstehen können. Als Risiken werden unzureichende diagnostische Fähigkeiten, Kommunikationsmängel, persönliche Probleme, der Therapie abträgliche Motive sowie die Verletzung ethischer Grundsätze verstanden. Andererseits sollen durch die SE die persönlichen Stärken und Potenziale weiterentwickelt werden.

In Österreich wird das Ziel der SE vom Gesetzgeber folgendermaßen definiert:

„Gegenstand dieses Verfahrens ist der Ausbildungskandidat selbst, der sich einer über mindestens $200 \mathrm{~h}$ gehenden Psychotherapie mit einer Lehrtherapeut*in einzeln oder in der Gruppe zu unterziehen hat. Dieser Ausbildungsprozess soll zu jener Persönlichkeitsveränderung führen, die die sachgemäße Handhabung der psychotherapeutischen Beziehung, einen Abbau von sogenannten ,blinden Flecken “ und 
Vorurteilen, eine Zunahme von Geduld, Toleranz und Empathiefähigkeit sowie einen verbesserten Umgang mit Arbeitsbelastungen erst ermöglichen“ (Kierein et al. 1991, S. 128).

Detaillierte Ziele für die Gruppen-SE und die Einzel-SE in der Psychodramaausbildung wurden bislang nicht publiziert. Für den Universitätslehrgang für das psychotherapeutische Fachspezifikum in der methodenspezifischen Ausrichtung an der Universität Innsbruck wurden diese im Rahmen der Lehrveranstaltungsbeschreibung aufgelistet (Fürst 2014):

- Erhöhung des Selbstwertgefühls und der sozialen Kompetenz

- Entwicklung von Spontaneität und Kreativität

- Vergrößerung des Rollenrepertoires

- Verbesserung der Selbst- und Fremdwahrnehmung

- Exploration von Rollen, der persönlichen Geschichte, dem Lebensplan, Verhaltensmustern und -strategien.

- Erhöhung der Motivation und Befähigung neues Verhalten zu entwerfen und zu erproben

- Vertiefung des zwischenmenschlichen Verstehens

- Erweiterung der Rollenflexibilität (Fähigkeit zur spontanen Übernahme verschiedenster Rollen)

- Erhöhung der persönlichen Belastbarkeit und Frustrationstoleranz

- Verbesserung der sprachlichen Umsetzung von Botschaften im Hinblick auf den Empfänger (Feedbacks)

- Vergrößerung der Reflexionsfähigkeit von Interaktionsprozessen im Psychodrama

- Einarbeitung in die Methode des Psychodramas und Beherrschung der Grundtechniken.

Inwieweit diese Ziele maßgeblich für den Therapieerfolg späterer Psychotherapeut*innen verantwortlich sind, lässt sich derzeit nur theoretisch begründen. Der empirische Beweis lässt noch auf sich warten.

\section{Qualitätssicherung}

Bezugnehmend auf Donabedian (1980), stellen Nodop und Strauß (2016) drei Aspekte der Qualitätssicherung in den Mittelpunkt: Strukturqualität, Prozessqualität und Ergebnisqualität.

\subsection{Strukturqualität}

Zur Strukturqualität zählen die Auswahl geeigneter Leiter*innen für die SE, sowie „Setting, Umfang, Frequenz und die zeitliche Anordnung innerhalb der Ausbildung und das Vorhandensein eines einheitlichen Konzepts für die SE“ (Nodop und Strauß 2016, S. $58 \mathrm{f}$.).

In den Anfängen der Psychodramaausbildung wurde zwischen den Leiter*innen einer SE-Gruppe und einer Theoriegruppe nicht unterschieden. Erst in den Jahren nach in Kraft treten des Psychotherapiegesetzes, wurde in Österreich ein besonde- 
res Augenmerk auf die Auswahl der Leiter*innen in Bezug auf deren spezifische Qualifikation gelegt. Aber noch immer gibt es keine operationalisierten Qualitätsmerkmale, die SE-Gruppenleiter*innen von Theorievermittler*innen unterscheiden.

Eine spezielle Ausbildung für Lehrtherapeut*innen, die SE im Gruppen- oder im Einzelsetting anbieten, gibt es nur in wenigen Ausbildungseinrichtungen. Gesetzlich ist die Voraussetzung für diese Tätigkeit in Österreich nur in Form einer bestimmten praktischen Vorerfahrung umschrieben. Mindestens 5 Jahre psychotherapeutische Tätigkeit wird dafür vorausgesetzt.

In anderen europäischen Ausbildungseinrichtungen richten sich die Institute nach den Minimalvorgaben bzw. Empfehlungen der Federation of European Psychodrama Training Institutes (FEPTO), wenn sie dort als Mitglieder aufgenommen wurden. Andere orientieren sich an den finanziellen Möglichkeiten der Auszubildenden, nach deren Vorbildung bzw. nach ihrer persönlichen Einschätzung.

Sowohl Umfang als auch Frequenz sind den Vorerfahrungen und dem Gutdünken überlassen.

\subsection{Prozessqualität}

Nodop und Strauß (2016) gliedern die Prozessqualität in die Bereiche: Vorwissen der Auszubildenden, das Abstimmen der SE Inhalte auf die Ziele der SE und die persönlichen Bedürfnissee der Teilnehmer*innen, sowie die Schaffung einer guten Atmosphäre.

So bleibt es im Wesentlichen dem jeweiligen Leiter bzw. der Leiterin der SE überlassen, inwieweit die SE von den Kandidat*innen ausschließlich aus der Rolle der Protagonist*innen erlebt wird oder auch aus der Rolle der Leiter*innen. Ein einheitliches Konzept für die SE im Sinne einer Manualisierung widerspricht der prozessorientierten Vorgehensweise im Psychodrama.

In einigen Ausbildungseinrichtungen wird die Therapeut*innenrolle oder die s.g. „Probeleitung“ nur als „,̈berprüfungselement“" eingesetzt, um einzuschätzen, inwieweit die Auszubildenden in der Lage sind, diese Rolle in Grundzügen zu übernehmen. In manchen Instituten zählt die Übernahme der Leitungsfunktion zum Standard in der SE von Beginn an.

Auch die Reflexion in der Gruppe über Prozess und Interventionen der Leiter*innen findet weder in den SE-Gruppen noch in der Einzellehrtherapie standardmäßig statt.

Begründet wird die unterschiedliche Vorgangsweise vorrangig mit der eigenen Ausbildungssozialisation. Psychodramatiker*innen mit psychoanalytischer Basisausbildung tendieren eher dazu, dem Konzept der „Lehrtherapie“ zu folgen, indem die Auszubildenden zuerst die Rolle des „Patienten“ bzw. der „Patientin“ erfahren sollen und die ,Regression“ nicht dadurch verhindert werden soll, dass Auszubildende frühzeitig eine Metarolle einnehmen.

Gleichzeitig zeigen die Rückmeldungen von Auszubildenden, dass der Rollenwechsel von der Peer-Rolle zu Leiter*innen-Rolle nach zwei bis drei Jahren Regression in einer SE Gruppe manchen sehr schwer fällt. Auch Lehrtherapeut*innen nehmen den Widerstand der Auszubildenden wahr, die Leitungsfunktion zu über- 
nehmen, wenn diese zu Übungszwecken ihre Gruppenkolleg*innen in Protagonistenspielen anleiten sollen.

Die frühe Übernahme von Leitungsfunktionen bereits in den Anfängen der Selbsterfahrungsgruppe scheint einen Übergang zu erleichtern. Studien dazu fehlen allerdings völlig.

\subsection{Ergebnisqualität}

Der Entwicklungsstand der persönlichen Kompetenzen wird von Nodop und Strauß (2016) zu den Ergebnisqualitäten gezählt, ebenso wie die Zufriedenheit mit der SE und der subjektiv eingeschätzte persönliche und professionelle Nutzen.

Es stellt sich die Frage, inwieweit es notwendig und sinnvoll ist, den Entwicklungsstand der persönlichen Kompetenzen aus der Beobachterrolle zu beurteilen oder inwieweit eine Selbstreflexion z.B.: in Form eines Tagebuches ausreichend wäre.

In der Gruppenselbsterfahrung wurde in mehreren Ausbildungseinrichtungen an zwei zeitlichen Punkten der SE eine Beurteilung durch Gruppe und Leiter*innen und durch den Kandidaten bzw. die Kandidatin selbst durchgeführt, die zur Reflexion des bisher Erreichten in Bezug auf die formulierten Ziele dienen soll. Diese „Überprüfungen“, wie sie von den Auszubildenden genannt werden, werden jedoch mehrheitlich als belastend, von manchen auch als bereichernd wahrgenommen.

In der Einzel-SE bleibt diese Beurteilung den Therapeut*innen überlassen, die über eventuell zusätzlich notwendige Stunden gemeinsam mit den Auszubildenden entscheiden.

Die anonymisierte Evaluation der SE durch die Auszubildenden wurde von vielen Ausbildungseinrichtungen in den 90er-Jahren eingeführt, um die Qualität der Ausbildung zu verbessern.

\section{Effekte und Wirksamkeit}

Absolvent*innen von Psychotherapieausbildungen betrachten die Eigentherapie in mehreren Bereichen als bedeutsam für ihre Therapeut*innenkompetenz. Willutzki et al. (1997) stellen fest, dass die Eigentherapie zur Überzeugung beiträgt, über eine hohe therapeutische Kompetenz zu Verfügen.

Mehrere Studien weisen auf die Wirksamkeit und Wichtigkeit der SE als Ausbildungsschritt hin und sehen sie als ein wesentliches Element für die Entwicklung zum Psychotherapeuten bzw. zur Psychotherapeutin (Orlinsky et al. 2005; Gahleitner et al. 2015). Unter anderem wurde auch die Wirkweise der SE in der PsychodramaAusbildung untersucht (Fürst 2020; Ockert 2016; Schnabel und Reif 2016). Diese Untersuchungen sind jedoch bei Weitem nicht ausreichend, um angemessene Schlüsse zu ziehen. 


\subsection{Negative Effekte}

Im Wesentlichen sind drei Bereiche negativer Effekte sichtbar:

- Abhängigkeit durch Mehrfachfunktionen der Leiter*innen

- Gruppenkonflikte und destruktive Gruppendynamik

- Unangemessenes Verhalten und mangelnde Kompetenz der SE-Leiter*innen

Durch die Evaluation der SE in der Ausbildung werden auch negative Aspekte sichtbar. Funktionsüberschneidungen von Leiter*innen der SE-Gruppen bzw. Einzeltherapien gehören zu den am häufigsten genannten. Die Doppel- und Mehrfachrollen von Lehrtherapeut*innen reduziert die Bereitschaft der Kandidat*innen sich zu öffnen (Nodop und Strauß 2016). Besonders in kleinen Ausbildungseinrichtungen sind die Leiter*innen der SE häufig gleichzeitig die Leiter*innen der Theorie und Technikgruppen und der Supervision. Am Ende entscheiden sie auch über den positiven bzw. negativen Abschluss oder weiteren Auflagen.

Ungelöste oder unausgesprochene Konflikte zwischen Gruppenteilnehmer*innen reduzieren die Bereitschaft Persönliches zu teilen. Auch innerhalb einer Gruppe können schwierige Situationen entstehen, wenn Abhängigkeitsbeziehungen von Teilnehmer*innen außerhalb der Gruppe bestehen.

Die Beziehung zu den Lehrtherapeut*innen beeinflusst wesentlich den Entwicklungsprozess der Auszubildenden, im positiven, wie im negativen Sinn.

\section{Perspektiven und Empfehlungen}

In einem Forschungsgutachten (Nodop und Strauß 2016) wurden deutschlandweit in 173 anerkannten Ausbildungseinrichtungen verschiedener Modalitäten Daten mithilfe von Fragebögen von Auszubildenden sowie Lehrkräften erhoben, die auf die Qualität der SE in der Ausbildung abzielten. Als Resultat empfiehlt die Gutachtergruppe unter anderem mindestens 50h für Einzel-SE. Die SE sollte nicht später als ein halbes Jahr nach Ausbildungsbeginn starten und ausbildungsbegleitend sein.

Funktionsüberschneidungen sind auch bei kleineren Ausbildungseinrichtungen vermeidbar, indem mehrere Institute zusammenarbeiten und damit verhindert wird, dass Lehrtherapeut*innen, die Leiter*innen der SE waren, Prüfungsfunktion übernehmen. Dadurch kann die Bereitschaft der Auszubildenden sich zu öffnen, vergrößert werden. Eine Wahlfreiheit im Hinblick auf die Leiter*innen der SE sollte gegeben sein.

Auch in Bezug auf die Konzepte zur SE besteht Handlungsbedarf, im Besonderen in der Einzel-SE.

Die verpflichtende Einzel-SE erscheint besonders im Hinblick darauf, dass bestimmte Themen in der Gruppe ausgeklammert werden, mehr als sinnvoll. Außerdem zeigt die Praxis, dass viele Therapeut*innen, Berater*innen und Supervisor*innen mehr Stunden im Einzelsetting arbeiten als in der Gruppe. Den Kandidat*innen zu vermitteln, wie der Prozess in einem längeren Einzelsetting zu handhaben ist, erleichtert den Einstieg in die Praxis. 
Die Vermeidung von Funktionsüberschneidungen lässt sich bei der durchschnittlichen Größe der Ausbildungseinrichtungen nur durch Kooperationen bewerkstelligen, was in der Folge einen Diskurs zur Konzeptentwicklung in der SE aber auch anderer Curriculumsinhalte mit verschiedenen Ausbildungsanbietern miteinschließen würde.

In der Einzellehrtherapie bzw. Einzel-SE sollte dabei verstärkt auf die individuelle Entwicklung der Auszubildenden Rücksicht genommen werden. Gleichzeitig sollen die Studierenden den Prozess in einem Einzelsetting nicht nur selbst erfahren, sondern auch reflektieren und die angewandten Techniken lernen.

Psychodrama ist eine prozessorientierte Methode. So ist es durchaus möglich, dass in einer Psychotherapie mit Patient*innen bestimmte Themen nie angesprochen bzw. in Szene gesetzt werden. Die Ausbildung hingegen dient nicht der Reduktion psychischen Leidens, sondern der Entwicklung der Kompetenz einen Beruf auszuüben. Deshalb muss der Inhalt der SE in der Ausbildung zu psychosozialen Berufen die gesamte Breite der menschlichen Themen umfassen. Es darf keine Aspekte geben, die vermieden oder ausgeklammert werden.

Werden sie von den Auszubildenden nicht selbst angesprochen, ist es Aufgabe der Lehrtherapeut*innen für diese Themen zu erwärmen und damit den Handlungsund Erfahrungsspielraum zu erweitern. Dazu gehört die Reflexion der Beziehungen zu Menschen des sozialen Beziehungsnetzes, zu Besitz, Geld, Macht, Heimat/ Fremdheit, Abhängigkeit/Freiheit, Krankheit/Gesundheit, Gott/Religion, Genuss/ Verzicht, Körper, Geist, Gefühlen, Endlichkeit, Freiheit, Normen, Lebenssinn u.a. Auch die Auseinandersetzung mit persönlichen Ressourcen, Schwächen und Handlungsmustern sollte Teil der SE sein.

Die Überprüfung, inwieweit die Ziele der SE erreicht wurden, erfolgt in vielen Psychodrama-Ausbildungseinrichtungen in Form von Selbstbeurteilung und Einschätzung der Gruppenteilnehmer*in und der jeweiligen Leiter*in. Von dieser Beurteilung hängt der weitere Ausbildungsfortgang ab. Diese Abhängigkeit von Gruppenmitgliedern und Leiter*innen wird in diesem Schritt besonders deutlich und wird von den Auszubildenden auch belastend erlebt.

Folgt man der Argumentation von Merod (2016) in seiner Auseinandersetzung mit „Selbsterfahrung als Selektionsinstrument“, so wird deutlich, dass die SE sich von allen Ausbildungsschritten am wenigsten als Selektionsinstrument eignet. Die Einbeziehung der Peers in die Entscheidung ist dabei ebenso kontraproduktiv, wie jener der Leiter*innen.

Noch ist völlig unerforscht, welche Persönlichkeitsmerkmale zu ,schlechten Therapeut*innen“ oder zu so genannten ,toxic shrinks“ führen (Merod 2016). Auch die Frage, ob diese bereits vor der Ausbildung erkannt werden können, ist nach wie vor unbeantwortet. Es fehlen wissenschaftlich belegte Kriterien, nach denen Lehrtherapeut*innen entscheiden könnten.

Die SE von jeder Beurteilung zu befreien, erscheint in der Berücksichtigung aller Erfahrungen als ein wichtiger Schritt zu größerer Öffnungsbereitschaft der Teilnehmer*innen und Entlastung für die Gruppendynamik.

Beurteilung und Selektion soll aus Sicht von Institutsleiter*innen (Nodop und Strauß 2013) in erster Linie den Supervisor*innen und an zweiter Stelle der Ausbildungsleitung überlassen werden. 
Die Evaluation der SE sollte zur Selbstverständlichkeit werden, sowohl für die Gruppen-SE als auch für die Einzel-SE. Dazu eignen sich anonymisierte Fragebögen mit geschlossenen und offenen Fragen, die die grundsätzliche Zufriedenheit mit der SE, der Gruppe und den Leiter*innen abfragen, die Atmosphäre, aber auch hilfreiche und hinderliche Interventionen, sowie die Relevanz für die persönliche Weiterentwicklung und Praxis.

\section{Zusammenfassung}

Psychodrama ist ein ganzheitlicher Ansatz, in dem SE ein impliziter Teil des Verfahrens ist. SE kann deshalb auch in der Ausbildung nicht als separierter Ausbildungsschritt betrachtet werden. Dadurch unterscheidet sich SE in der Psychodramaausbildung von anderen Modalitäten unabhängig vom Format.

Die Erfahrung und das Lernen in Rollen und Interaktionen ist zentrales Element des Psychodramas und damit immanenter Bestandteil auch der Qualität der Ausbildung. SE ist nicht nur ein Ausbildungsschritt, sondern integriert in Theorievermittlung, Praxis und Supervision.

Gerade deshalb ist es wichtig die Konzeptentwicklung in Gruppen- und Einzelselbsterfahrung voranzutreiben. Die Bewusstheit, dass Rollen- und Funktionsüberschneidungen Konflikte mit sich bringen, sollte besonders in der Ausbildung dazu führen, dass diese vermieden werden.

Forschungsergebnisse, aber auch guter Hausverstand, legen nahe, dass sich SE nicht als Selektionsinstrument eignet. Auszubildenden soll die Möglichkeit gegeben werden, angstfrei alle Lebensthemen zu explorieren, ohne am Ende einer Beurteilung ausgesetzt zu werden. Wissen und Kompetenzen der Kandidat*innen sollten von Supervisor*innen und Ausbildungsleiter*innen beurteilt werden, solange nicht gesichert ist, welche Persönlichkeitsmerkmale einen „guten“ von einem „schädigenden“" Therapeuten bzw. einer Therapeutin unterscheiden. Letztendlich darf nicht außer Acht gelassen werden, dass die Qualität der SE durch anonymisierte Evaluation der Ausbildungsteile und durch die Supervision der Lehrtherapeut*innen verbessert werden kann.

Funding Open access funding provided by Sigmund Freud Privatuniversität Wien.

Open Access Dieser Artikel wird unter der Creative Commons Namensnennung 4.0 International Lizenz veröffentlicht, welche die Nutzung, Vervielfältigung, Bearbeitung, Verbreitung und Wiedergabe in jeglichem Medium und Format erlaubt, sofern Sie den/die ursprünglichen Autor(en) und die Quelle ordnungsgemäß nennen, einen Link zur Creative Commons Lizenz beifügen und angeben, ob Änderungen vorgenommen wurden.

Die in diesem Artikel enthaltenen Bilder und sonstiges Drittmaterial unterliegen ebenfalls der genannten Creative Commons Lizenz, sofern sich aus der Abbildungslegende nichts anderes ergibt. Sofern das betreffende Material nicht unter der genannten Creative Commons Lizenz steht und die betreffende Handlung nicht nach gesetzlichen Vorschriften erlaubt ist, ist für die oben aufgeführten Weiterverwendungen des Materials die Einwilligung des jeweiligen Rechteinhabers einzuholen.

Weitere Details zur Lizenz entnehmen Sie bitte der Lizenzinformation auf http://creativecommons.org/ licenses/by/4.0/deed.de. 


\section{Literatur}

Balint, E., \& Balint, M. (1955). Dynamics of trainings in groups for psychotherapy. British Journal of Medical Psychology, 28, 135-143.

Bender, J. (2003). Entdeckendes Lernen - Entstehung, notwendige Voraussetzung und Kompetenzen, Praxis. [Studienarbeit] (GRIN)

Bergin, A.E., \& Garfield, S.L. (1971). Handbook of psychotherapy and behavior change. An empirical analysis. New York: Wiley.

Buer, F. (1999). Morenos therapeutische Philosophie. Zu den Grundideen von Psychodrama und Soziometrie (3. Aufl.). Wiesbaden: VS. https://doi.org/10.1007/978-3-322-97414-3.

Buer, F. (2010). Psychodrama und Gesellschaft. Wiesbaden: VS Verlag für Sozialwissenschaften.

Donabedian, A. (1980). Explorations in quality assessment and monitoring. Ann Arbor: Health Administration Press.

Fontaine, P. (1994). Study and coordination of psychodrama education in Europe. Leuven: Eigenverlag. Preliminary documents

Foulkes, S.H., \& Anthony, E. J. (1984). Group psychotherapy. The psychoanalytic approach (2. Aufl.). London: H. Karnac (Books) (Maresfield library).

Fürst, J. (2020). Role development in psychodrama training-Findings and challenges. Zeitschrift Für Psychodrama und Soziometrie. Psychodrama. Empirical Research and Science, 19, 239-253.

Fürst, J. (2014). Lehrveranstaltungen: Gruppenselbsterfahrung. https://lms.uibk.ac.at/auth/RepositoryEntry/ 429719552/CourseNode/84028182960823. Zugegriffen: 31. Mai 2021.

Gahleitner, S., Gerlich, K., \& Liegl, G. (2015). Selbsterfahrung als Ausbildungsbestandteil der Psychotherapie - Qualitätsmerkmal oder Mythos? Zentrale Ergebnisse des Forschungsprojektes „Die Rolle der Selbsterfahrung in der Psychotherapieausbildung“. http://www.resonanzen-journal.org. Zugegriffen: 2. Juli 2021. zuletzt aktualisiert am 15.11.2015.

Hutter, C., \& Schwehm, H. (Hrsg.). (2009). J. L. Morenos Werk in Schlüsselbegriffen (1. Aufl.). Wiesbaden: VS.

Kierein, M., Pritz, A., \& Sonneck, G. (1991). Psychologengesetz, Psychotherapiegesetz. Kurzkommentar. Wien: Orac.

Korbei, L. (1996). Zur Idee der Selbsterfahrung in der Ausbildung zum/zur klientenzentrierten Psychotherapeuten/Psychotherapeutin. Psychotherapie Forum, 4/4 203-209.

Krall, H., Fürst, J., \& Fontaine, P. (Hrsg.). (2013). Supervision in psychodrama. Experiential learning in psychotherapy and training. Wiesbaden: Springer VS.

Leutz, G. (1974). Psychodrama. Theorie und Praxis. Berlin: Springer.

Merod, R. (2016). Selbsterfahrung (SE) als Mittel der Qualitätskontrolle. In T. Mösler, S. Poppek, J.C. Kemper, A. Rose \& W. Dorrmann (Hrsg.), Der Blick auf sich selbst. Selbsterfahrung in der Psychotherapie. Tübingen: Psychotherapie-Verlag.

Moreno, J.L. (1945). Scientific foundations of group psychotherapy. Sociometry, 8, 77-84.

Moreno, J.L. (1953). How Kurt Lewin's “Research Center for Group Dynamics” started. Sociometry, 16, 101-104.

Moreno, J.L. (1974). Die Grundlagen der Soziometrie. Wege zur Neuordnung der Gesellschaft (3. Aufl.). Opladen: Westdeutscher Verlag. Unter Mitarbeit von G.A. Leutz

Moreno, J. L., \& Toeman, Z. (1942). The group approach in psychodrama. Sociometry, 5, 191-195.

Mösler, T., Poppek, S., Kemper, J. C., Rose, A., \& Dorrmann, W. (Hrsg.). (2016). Der Blick auf sich selbst. Selbsterfahrung in der Psychotherapie. Tübingen: Psychotherapie-Verlag.

Nodop, S., \& Strauß, B. (2016). Qualitätsaspekte der Selbsterfahrung in der psychotherapeutischen Ausbildung in Deutschland. Quantitative und qualitative Ergebnisse des Forschungsgutachtens. In T. Mösler, S. Poppek, J. C. Kemper, A. Rose \& W. Dorrmann (Hrsg.), Der Blick auf sich selbst. Selbsterfahrung in der Psychotherapie (S. 56-88). Tübingen: Psychotherapie-Verlag.

Ockert, F. (2016). Selbsterfahrung im Psychodrama. Individuelle Erlebniswelten im gruppentherapeutischen. Hamburg: Universität Hamburg. Institut für Psychologie

Orlinsky, D.E., Geller, J.D., \& Norcross, J.C. (Hrsg.). (2005). The psychotherapist's own psychotherapy. Patient and clinician perspectives. Oxford, New York: Oxford University Press.

Rogers, C. (1977). Entwicklung und gegenwärtiger Stand meiner Ansichten über zwischenmenschliche Beziehungen. In Gesellschaft für wissenschaftliche Gesprächspsychotherapie (Hrsg.), Die klientenzentrierte Gesprächspsychotherapie (S. 11-24). München: Kindler.

Schmelzer, D., \& Hirsch, R. (2000). Supervision und Selbsterfahrung. In W. Senf, M. Broda \& G. Amann (Hrsg.), Praxis der Psychotherapie. Ein integratives Lehrbuch: Psychoanalyse, Verhaltenstherapie, systemische Therapie (2. Aufl. S. 363-371). Stuttgart: Thieme. 
Schnabel, K., \& Reif, J. (2016). Wege zur Evaluation psychodramatischer Ausbildungen. Zeitschrift Für Psychodrama und Soziometrie. Psychodrama. Empirical Research and Science, 2, 289-296.

Scholkmann, A. (2016). Forschend-entdeckendes Lernen. (Wieder-)Entdeckung eines didaktischen Prinzips. In B. Berendt, A. Fleischmann, N. Schaper, B. Szczyrba, M. Wiemer \& J. Wildt (Hrsg.), Handbuch: Hochschullehre (S. 1-36). Berlin: DUZ Verlags- und Medienhaus.

Schön, K. (2001). Selbsterfahrung in der Verhaltenstherapie-Ausbildung: Prozess- und Ergebnisqualität aus Sicht der Teilnehmer/innen. http://bibd.uni-giessen.de/gdoc/2002/uni/d020056.pdf. Zugegriffen: 28. Apr. 2021.

Tschuschke, V. (2016). Widerstand gegen die Selbsterfahrung in der Psychotherapie. In T. Mösler, S. Poppek, J.C. Kemper, A. Rose \& W. Dorrmann (Hrsg.), Der Blick auf sich selbst. Selbsterfahrung in der Psychotherapie (S. 89-100). Tübingen: Psychotherapie-Verlag.

Willutzki, U., Ambühl, H., Cierpka, M., Meyerberg, J., Orlinsky, D., \& das SPR Collaborative Research Network (1997). Zufrieden oder ausgebrannt: Die berufliche Moral von Psychotherapeuteinnen und Psychotherapeuten. In P.L. Janssen, M. Cierpka \& P. Buchheim (Hrsg.), Psychotherapie als Beruf (S. 207-222). Göttingen: Vandenhoeck \& Ruprecht.

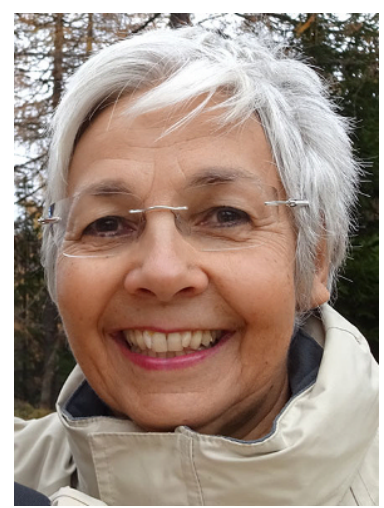

Jutta Fürst Dr., Klinische Psychologin und Psychotherapeutin für Psychodrama und Katathym Imaginative Psychotherapie. Lehrtherapeutin, Supervisorin und Coach. Langjährige private psychotherapeutische Praxis und Lehrtätigkeit an verschiedenen Universitäten. 20 Jahre wissenschaftliche Leiterin des Universitätslehrganges für das psychotherapeutische Fachspezifikum in der Methode Psychodrama an der Universität Innsbruck. Mitbegründerin, Vorstandsmitglied und ehemalige Präsidentin der Federation of European Psychodrama Training Organisation (FEPTO). 